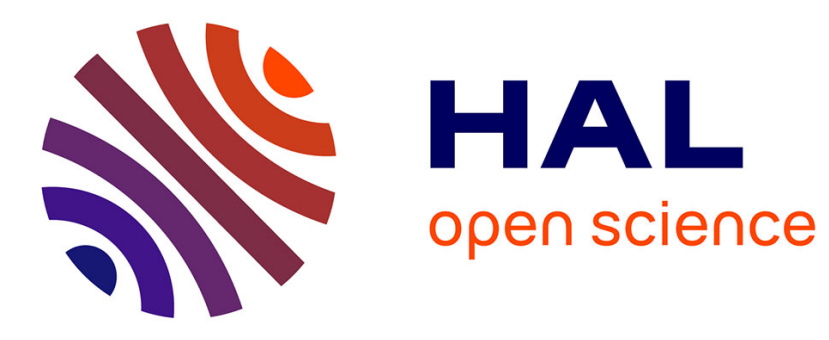

\title{
Rôle du temps d'insolation dans les performances lithographiques du système Ag2Se/GeSey
}

\author{
Y. Brocheton, B. Cros, A. Tissier, M. Ribes
}

\section{To cite this version:}

Y. Brocheton, B. Cros, A. Tissier, M. Ribes. Rôle du temps d'insolation dans les performances lithographiques du système Ag2Se/GeSey. Revue de Physique Appliquée, 1989, 24 (12), pp.11291136. 10.1051/rphysap:0198900240120112900 . jpa-00246150

\section{HAL Id: jpa-00246150 https://hal.science/jpa-00246150}

Submitted on 1 Jan 1989

HAL is a multi-disciplinary open access archive for the deposit and dissemination of scientific research documents, whether they are published or not. The documents may come from teaching and research institutions in France or abroad, or from public or private research centers.
L'archive ouverte pluridisciplinaire HAL, est destinée au dépôt et à la diffusion de documents scientifiques de niveau recherche, publiés ou non, émanant des établissements d'enseignement et de recherche français ou étrangers, des laboratoires publics ou privés. 
Classification

Physics Abstracts

$42.70 \mathrm{G}$

\title{
Rôle du temps d'insolation dans les performances lithographiques du système $\mathrm{Ag}_{2} \mathrm{Se} / \mathrm{GeSe}_{y}$
}

\author{
Y. Brocheton $\left({ }^{1}\right)$, B. Cros $\left({ }^{2}\right)$, A. Tissier $\left({ }^{1}\right)$ et M. Ribes $\left({ }^{2}\right)$ \\ (1) CNET-Grenoble, BP 98, chemin du Vieux-Chêne, 38243 Meylan, France \\ ( ${ }^{2}$ Laboratoire de Physicochimie des Matériaux Solides, U.R.A. D0407, USTL, Place Eugène Bataillon, \\ 34060 Montpellier Cedex 1, France
}

(Reçu le 10 juillet 1989, révisé et accepté le 7 septembre 1989)

\begin{abstract}
Résumé. - Les phénomènes associés à la migration des ions argent et les modèles de formation de l'image dans le système $\mathrm{Ag}_{2} \mathrm{Se} / \mathrm{GeSe}_{y}$ sont discutés. L'étude du rôle du temps d'insolation dans les performances lithographiques du système montre qu'à dose constante, un abaissement de l'intensité incidente améliore la sélectivité, tandis que le contraste et la résolution sont peu modifiés. L'utilisation d'un faible débit de dose s'accompagne d'une élévation des doses seuils (doses au-delà desquelles les motifs résistent au développement) et d'une perte de contrôle accentuée. Ces résultats sont confirmés par une simulation estimant la variation globale des largeurs de traits en fonction de la dose d'exposition.
\end{abstract}

\begin{abstract}
Phenomena related with the silver ions migration and models of image formation in the system $\mathrm{Ag}_{2} \mathrm{Se} / \mathrm{GeSe}_{y}$ are discussed. The role of exposure time on the lithographic capabilities of the system has been studied : increasing the.incident intensity at constant dose improves the selectivity of the system, whereas the contrast and the resolution are not much modified. The use of a weak exposure dose is accompanied by an increase of the threshold doses and a pronounced loss of control. These results are corroborated by a simulation wich gives an estimation of the whole variation of the linewidths as a function of the exposure dose.
\end{abstract}

\section{Introduction.}

Les résines inorganiques sont des matériaux prometteurs pour des applications en microlithographie [1]. Le procédé lithographique est basé sur le phénomène de la migration photoinduite de l'argent ou photodopage. La formation de l'image latente dans le système $\mathrm{Ag}_{2} \mathrm{Se} / \mathrm{GeSe}_{y}$ fait intervenir des mécanismes spécifiques. Ils reposent sur les mouvements verticaux et horizontaux (photodopage et diffusion latérale) des ions argent dans la couche active lors de l'insolation. L'importance relative accordée à l'une ou l'autre de ces migrations aboutit à des interprétations différentes des performances lithographiques observées. Si au niveau de la résolution limite les dimensions annoncées sont comparables, les pertes de contrôle dimensionnel diffèrent sensiblement avec les auteurs.

Tai et al. développent un modèle dans lequel la taille finale des motifs est régie par le photodopage. $\mathrm{Ce}$ dernier engendre les phénomènes de photoblanchiment et de bord abrupt [2-6]. Il est responsable des effets de proximité. Leurs travaux démontrent la possibilité de reproduire sans variation notable de côtes des structures diversement entourées (ligne isolée, espace isolé, motifs $\mathrm{L} / \mathrm{S}$ soit une succession périodique de lignes et d'espaces d'égales dimensions) pour une même dose d'insolation. Ils constatent une tolérance d'exposition d'environ $20 \%$ respectant la largeur des motifs. Elle se traduit par l'apparition d'un plateau dans les courbes de variations dimensionnelles en fonction du temps d'exposition. Son existence, attribuée au phénomène de bord abrupt, est mise à profit lors de la reproduction simultanée de motifs submicroniques $(0,5 \mu \mathrm{m} \mathrm{L} / \mathrm{S})$ et nettement microniques $(5 \mu \mathrm{m} \mathrm{L} / \mathrm{S})$. Une exacte correspondance entre la dimension après développement et celle de l'ouverture sur le masque est obtenue. Par contre, Utsugi et ses collaborateurs développent un concept mettant l'accent sur la diffusion latérale. Ils constatent que lors d'insolations plus brèves, aucune tolérance d'exposition n'apparaît. Ils soulignent que la largeur des motifs s'accroît continuellement avec la dose d'exposition 
et d'autant plus rapidement que la taille nominale à reproduire est faible. Ils interprètent cette tendance par des « changements topologiques de surface sous irradiation » $[7,8]$.

Ces différences majeures relevées par les auteurs au niveau des performances du système, qui sont interprétées à l'aide de deux modèles de formation de l'image, mettent en valeur le rôle prépondérant du temps d'insolation. Ce dernier conditionne aussi, selon les critères développés par Leung et al. [9-11], l'apparition des phénomènes induits par le photodopage au travers de la longueur de diffusion des ions argent dans la couche de sensibilisation. C'est pourquoi nous avons entrepris une étude du rôle du temps d'insolation sur les performances lithographiques du système afin de les améliorer.

\section{Analyse des modèles.}

Les modèles proposés nécessitent quelques commentaires. L'interprétation des performances lithographiques basée sur le phénomène de «changement topologique photoassisté de la surface» souffre de graves lacunes. En premier lieu, les auteurs ne font pas mention de l'évolution des collines après désensibilisation. Or cette étape est importante car la stabilité des îlots doit être altérée lors du retrait de l'argent non photodopé des parties non irradiées. Une seconde remarque porte sur la formation même de telles accumulations. En effet, la diffusion latérale ne peut provenir que d'un gradient de concentration créé dans la couche de sensibilisation. Seule la déplétion de cette couche par le photodopage permet d'expliquer son existence. Pour ces raisons, nous pensons que le photodopage détermine la dimension finale des motifs et que ce phénomène n'en est qu'une manifestation parallèle.

Si l'on considère le modèle de Tai, dans les conditions usuelles d'emploi du système (couche de sensibilisation relativement mince), seuls les phénomènes d'amplification dépendant du motif et le phénomène de bord abrupt apparaissent. Ce dernier, dont l'existence est confirmée par plusieurs équipes opérant avec des couches de sensibilisation de structure différente $[2,3,12-15]$, a fourni la première raison de la haute résolution du système. La condition de taille minimale imposée par les critères d'apparition des mécanismes associés à la migration des ions argent limite cependant le phénomène de bord abrupt.

Nous allons voir que les différents modèles sont compatibles avec une conception basée sur l'environnement des motifs.

\section{Résultats expérimentaux.}

Dans cette étude, nous nous sommes limités aux performances du système $\mathrm{Ag}_{2} \mathrm{Se} / \mathrm{GeSe}_{5,5}$ dans des conditions d'utilisation se rapprochant d'un environ- nement industriel où les temps d'insolation souhaités sont nécessairement inférieurs à quelques secondes par puce. Notre discussion portera sur l'influence de la diffusion latérale sur le contraste du procédé et sur les tolérances d'exposition.

3.1 CONTRASTE. - Les plaquettes sont insolées sur un photorépéteur (Perkin-Elmer SRA 100) délivrant une intensité maximale de $0,5 \mathrm{~W} / \mathrm{cm}^{2}$. Le film de chalcogénure, de $210 \mathrm{~nm}$ d'épaisseur initiale, est sensibilisé par immersion dans une solution de nitrate d'argent $(0,1 \mathrm{M})$ pendant 180 secondes. La mesure par rétrodiffusion Rutherford (RBS) de l'épaisseur de la couche de séléniure d'argent formée est de $6,5 \mathrm{~nm}$. Les doses d'exposition vont de 50 à $500 \mathrm{~mJ} / \mathrm{cm}^{2}$ pour les trois intensités incidentes utilisées $\left(122 \mathrm{~mW} / \mathrm{cm}^{2}, 245 \mathrm{~mW} / \mathrm{cm}^{2}\right.$ et $\left.304 \mathrm{~mW} / \mathrm{cm}^{2}\right)$. Elles sont obtenues en faisant varier les temps d'exposition. La longueur de diffusion des atomes d'argent dans la couche de sensibilisation correspondante est donnée dans le tableau I (elle est déterminée en utilisant la formule simple $L=(D \cdot t)^{1 / 2}$ avec $D=1 \mu \mathrm{m} . \mathrm{cm}^{-2}$ ).

Tableau I. - Longueurs de diffusion associées aux trois intensités incidentes.

[Diffusion lengthes associated with the three incident intensities.]

\begin{tabular}{|c|c|c|c|c|c|c|}
\hline $\begin{array}{c}\text { Dose } \\
\left(\mathrm{mJ} / \mathrm{cm}^{2}\right)\end{array}$ & \multicolumn{2}{|l|}{$I_{0}=122 \mathrm{~mW} / \mathrm{cm}^{2}$} & \multicolumn{2}{|l|}{$I_{0}=245 \mathrm{~mW} / \mathrm{cm}^{2}$} & \multicolumn{2}{|l|}{$I_{0}=304 \mathrm{~mW} / \mathrm{cm}^{2}$} \\
\hline & $\begin{array}{c}\text { temps } \\
\text { d'expo- } \\
\text { sition } \\
(\mathrm{s})\end{array}$ & $L(\mu \mathrm{m})$ & $\begin{array}{c}\text { temps } \\
\text { d'expo- } \\
\text { sition } \\
(\mathrm{s})\end{array}$ & $L(\mu \mathrm{m})$ & $\begin{array}{c}\text { temps } \\
\text { d'expo- } \\
\text { sition } \\
(\mathrm{s})\end{array}$ & \\
& & & & $(\mu \mathrm{m})$ \\
& 0,41 & 0,64 & 0,20 & 0,45 & 0,16 & 0,40 \\
50 & 0,82 & 0,91 & 0,40 & 0,64 & 0,33 & 0,57 \\
100 & 1,23 & 1,11 & 0,61 & 0,78 & 0,49 & 0,70 \\
150 & 1,64 & 1,28 & 0,81 & 0,90 & 0,66 & 0,81 \\
200 & 2,05 & 1,43 & 1,01 & 1,00 & 0,82 & 0,91 \\
250 & 2,05 & 1,57 & 1,22 & 1,10 & 0,98 & 0,99 \\
300 & 2,46 & 1,57 & 1,15 & 1,07 \\
350 & 2,87 & 1,70 & 1,42 & 1,19 & 1,15 \\
400 & 3,28 & 1,82 & 1,62 & 1,87 & 1,31 & 1,15 \\
450 & 3,69 & 1,92 & 1,82 & 1,35 & 1,48 & 1,21 \\
500 & 4,09 & 2,03 & 2,02 & 1,42 & 1,61 & 1,28 \\
\hline
\end{tabular}

Le contraste est représenté par la pente au point milieu de la courbe donnant les épaisseurs résiduelles en fonction de la dose d'insolation. Elles ont été mesurées à l'aide de la pointe de $0,2 \mu \mathrm{m}$ d'un palpeur mécanique sur des plots de $10 \mu \mathrm{m}$ après deux minutes de développement dans une solution d'hydroxyde de tétraméthylammonium (TMAH) $0,1 \mathrm{M}$ et $\mathrm{Na}_{2} \mathrm{~S} 0,05 \mathrm{M}$. Les mesures portent sur plusieurs puces et les figures sont présentées sous forme de nuages de points (Figs. 1a-1c). Les valeurs 


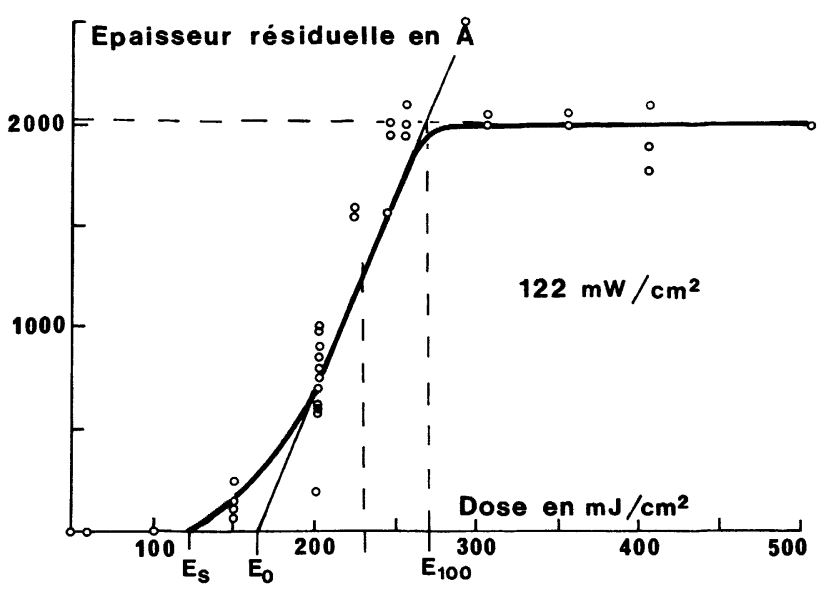

a)

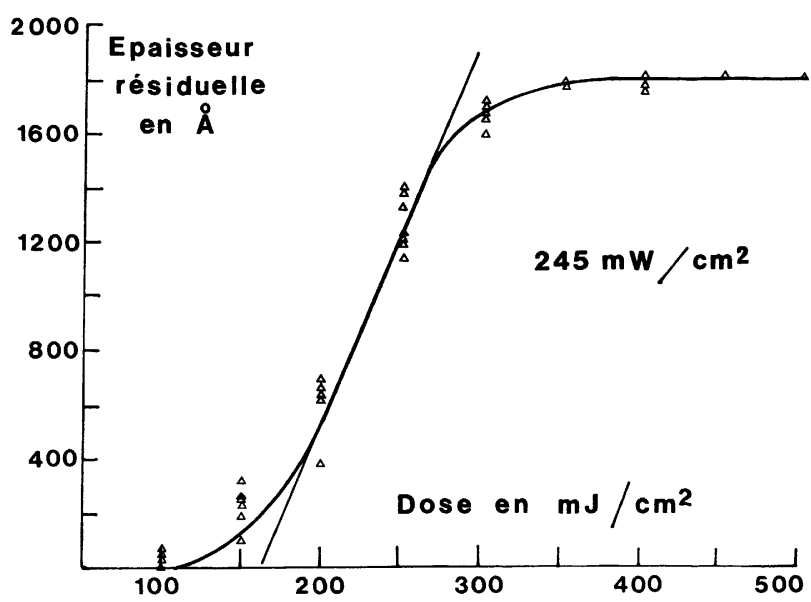

b)

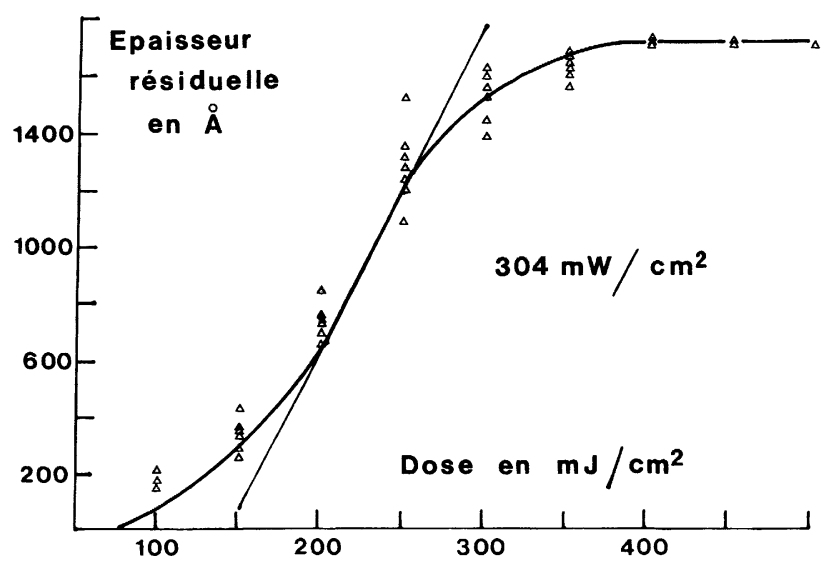

c)

Fig. 1. - Epaisseur résiduelle après développement de la couche de chalcogénure en fonction de la dose d'insolation. a) Intensité incidente $122 \mathrm{~mW} / \mathrm{cm}^{2}$; b) Intensité incidente $245 \mathrm{~mW} / \mathrm{cm}^{2}$; c) Intensité incidente $304 \mathrm{~mW} / \mathrm{cm}^{2}$.

[Remaining thickness after development as a function of the exposure dose.] représentatives qui en sont extraites sont consignées dans le tableau II. Ce sont (Fig. 1a) :

- l'épaisseur maximale résiduelle après développement qui permet de calculer la sélectivité du système ;

- la dose correspondant au point milieu donnant sa sensibilité ;

- la dose maximale d'insolation $E_{0}$ correspondant à un motif totalement développé ;

- la dose minimale correspondant à un motif non dégradé $E_{100}$ (ces deux dernières données permettent le calcul du contraste qui détermine le pouvoir de résolution du système);

- la dose seuil $E_{\mathrm{s}}$ qui correspond à la dose permettant de conserver une épaisseur minimale après développement.

\section{Discussion :}

Le débit de dose affecte principalement la sélectivité du système. Ceci a des répercussions sur la résolution et sur les doses seuils.

- La sélectivité varie de 5,25 à 21 lorsque l'intensité incidente est abaissée de $304 \mathrm{~mW} / \mathrm{cm}^{2}$ à $122 \mathrm{~mW} / \mathrm{cm}^{2}$. Pour cette dernière intensité, elle se situe à un niveau comparable à celui qui est rapporté dans la littérature [16-18] pour un développement par voie humide (Tab. III). Cet abaissement de sélectivité est imputable à une variation de photodopage. Le motif résiste d'autant plus longtemps au développement qu'une quantité importante d'argent est photodopée pour assurer la protection de sa surface. A faible dose, la saturation n'étant pas atteinte, le photodopage est limité par l'absorptivité de la couche de sensibilisation. Celle-ci atténue l'intensité lumineuse d'environ $6 \%$. Cette valeur relativement faible est due à son épaisseur. Cette barrière au photodopage est d'autant plus efficace que l'intensité incidente est basse $\left(122 \mathrm{~mW} / \mathrm{cm}^{2}\right)$. Dans ce cas, la quantité d'argent photodopé diminue ; ceci se traduit par une élévation de la dose seuil par rapport à une insolation effectuée brièvement avec une source intense $\left(304 \mathrm{~mW} / \mathrm{cm}^{2}\right)$. A dose élevée, le meilleur maintien des motifs provient de l'apport extérieur d'atomes d'argent. Pour les fortes intensités, la longueur de diffusion de l'argent est d'environ un micron. Le surplus d'atomes photodopés est incorporé à la périphérie des motifs. Le phénomène de bord abrupt peut contribuer néanmoins à une meilleure tenue des motifs au développement. Par contre, lorsque les doses sont obtenues en allongeant le temps d'insolation (pour $\left.122 \mathrm{~mW} / \mathrm{cm}^{2}\right)$, l'argent migre au-delà de la zone floue $(L \simeq 2 \mu \mathrm{m})$. La conséquence est une augmentation de la quantité d'argent disponible au centre du motif se traduisant par une élévation de la sélectivité et un abaissement de la dose respectant son intégrité.

- Le constraste est légèrement amélioré par une diminution du débit de dose. Il varie de 3,24 pour 
Tableau II. - Données extraites des figures $1 a, b, c$.

[Datas deduced from 1a, b, c figures.]

\begin{tabular}{|c|c|c|c|c|c|c|c|c|c|c|}
\hline $\begin{array}{c}\text { Intensité } \\
\left(\mathrm{mW} / \mathrm{cm}^{2}\right)\end{array}$ & $\begin{array}{c}\text { Epaisseur } \\
\text { résiduelle } \\
(\AA)\end{array}$ & Sélectivité & $\begin{array}{c}\text { Sensibilité } \\
\left(\mathrm{mJ} / \mathrm{cm}^{2}\right)\end{array}$ & $\begin{array}{c}\text { Dose } \\
\text { seuil } E_{\mathrm{s}} \\
\left(\mathrm{mJ} / \mathrm{cm}^{2}\right)\end{array}$ & $\begin{array}{c}E_{0} \\
\left(\mathrm{~mJ}^{\prime} \mathrm{cm}^{2}\right)\end{array}$ & $\begin{array}{c}E_{100} \\
\left(\mathrm{~mJ} / \mathrm{cm}^{2}\right)\end{array}$ & Contraste & $\begin{array}{c}\text { MTF } \\
R_{\mathrm{p}}(\text { paires } \\
\text { de lignes } \\
\text { par } \mathrm{mm})\end{array}$ & $\begin{array}{c}R_{\mathrm{p}} \\
(\mu \mathrm{m})\end{array}$ \\
\hline 122 & 2000 & 21 & 215 & $120-130$ & 165 & 260 & 5,06 & 0,223 & 930 & 0,54 \\
247 & 1800 & 7 & 225 & 100 & 160 & 290 & 3,76 & 0,296 & 880 & 0,57 \\
304 & 1700 & 5,25 & 220 & $70-80$ & 145 & 295 & 3,24 & 0,341 & 720 & 0,59 \\
\hline
\end{tabular}

une intensité de $304 \mathrm{~mW} / \mathrm{cm}^{2}$ à 5,06 pour $122 \mathrm{~mW} / \mathrm{cm}^{2}$. Cette variation de contraste n'est cependant pas de nature à affecter sensiblement la résolution du système. Le pouvoir de résolution évolue de $0,59 \mu \mathrm{m}$ à $0,54 \mu \mathrm{m}$. Il se situe aux alentours de $0,6 \mu \mathrm{m}$ quelle que soit l'intensité incidente. Cette valeur est comparable aux données bibliographiques (Tab. III) et seul un développe'ment par voie sèche permettra d'atteindre les résolutions ultimes.

- La sensibilité évolue peu avec le débit de dose. Elle demeure d'environ $220 \mathrm{~mJ} / \mathrm{cm}^{2}$. Le système est donc environ deux fois moins sensible qu'une résine organique $\left(110 \mathrm{~mJ} / \mathrm{cm}^{2}\right)$. Par contre, la pénalité la plus sérieuse provient des temps d'exposition. Les meilleures performances du système sont obtenues pour les intensités incidentes faibles. En effet, favoriser la migration des atomes d'argent dans la couche de sensibilisation implique une exposition prolongée. Ce facteur peut s'avérer limitatif d'un point de vue des cadences industrielles.

- La dose seuil représente la sensibilité effective de la résine au processus de photodopage. Elle permet d'estimer à partir de quelle dose une quantité d'argent suffisante aura migré à l'intérieur du dépôt pour qu'une épaisseur quelconque puisse subsister lors du développement. A cause de l'absorption de la couche de sensibilisation, elle évolue de $70-80 \mathrm{~mJ} / \mathrm{cm}^{2}$ à $120-130 \mathrm{~mJ} / \mathrm{cm}^{2}$ lorsque l'intensité est abaissée de $304 \mathrm{~mW} / \mathrm{cm}^{2}$ à $122 \mathrm{~mW} / \mathrm{cm}^{2}$. Comme nous allons le voir, cette augmentation du seuil de sensibilité n'est pas sans incidence sur le contrôle dimensionnel.

Tableau III. - Méthodes de développement disponibles pour le système $\mathrm{Ag}_{2} \mathrm{Se} / \mathrm{GeSe}_{y}$.

[Development methods available for the $\mathrm{Ag}_{2} \mathrm{Se} / \mathrm{GeSe}_{y}$ system.]

\begin{tabular}{|c|c|c|c|c|c|}
\hline $\begin{array}{c}\text { Dévelop- } \\
\text { pement }\end{array}$ & $\begin{array}{c}\text { Sélec- } \\
\text { tivité }\end{array}$ & Contraste & $\begin{array}{c}R_{\mathrm{p}} \\
(\mu \mathrm{m})\end{array}$ & $\begin{array}{c}\text { Sensibilité } \\
\left(\mathrm{mJ} / \mathrm{cm}^{2}\right)\end{array}$ & Réf. \\
\hline $\begin{array}{c}\text { humide } \\
\mathrm{CF}_{4}\end{array}$ & 20 & 4 & 0,55 & $200-700$ & $16-18$ \\
$\mathrm{SF}_{6}$ & 500 & 4 & 0,55 & 90 & 19 \\
$4-8$ & 0.5 & $40-50$ & 20 \\
\hline
\end{tabular}

3.2 Contrôle DE DIMENSION. - Parallèlement, les tolérances d'exposition ont été estimées par la mesure des variations de dimensions de quatre motifs tests périodiques de dimensions croissantes $(0,7 \mu \mathrm{m}, 1 \mu \mathrm{m}, 1,6 \mu \mathrm{m}$ et $2 \mu \mathrm{m} \mathrm{L} / \mathrm{S})$. La largeur des motifs est mesurée en microscopie électronique à balayage. Six mesures ont été effectuées par motif. L'écart-type est de $0,22 \mu \mathrm{m}$. Signalons, d'ores et déjà, que les résultats obtenus pour les dimensions les plus restreintes $(1 \mu \mathrm{m}$ et $0,7 \mu \mathrm{m})$, très altérées lors du développement, sont les moins fiables.

Les valeurs expérimentales sont reportées sur la figure 2. La variation de dimension est explorée entre $150 \mathrm{~mJ} / \mathrm{cm}^{2}$ et $450 \mathrm{~mJ} / \mathrm{cm}^{2}$ pour deux intensités différentes $\left(122 \mathrm{~mW} / \mathrm{cm}^{2}\right.$ et $\left.304 \mathrm{~mW} / \mathrm{cm}^{2}\right)$. Le graphique permet d'obtenir :

- la dose nominale correspondant à chaque taille de motif (c'est la dose nécessaire pour que la dimension du motif développé corresponde à celle de l'ouverture sur le masque);

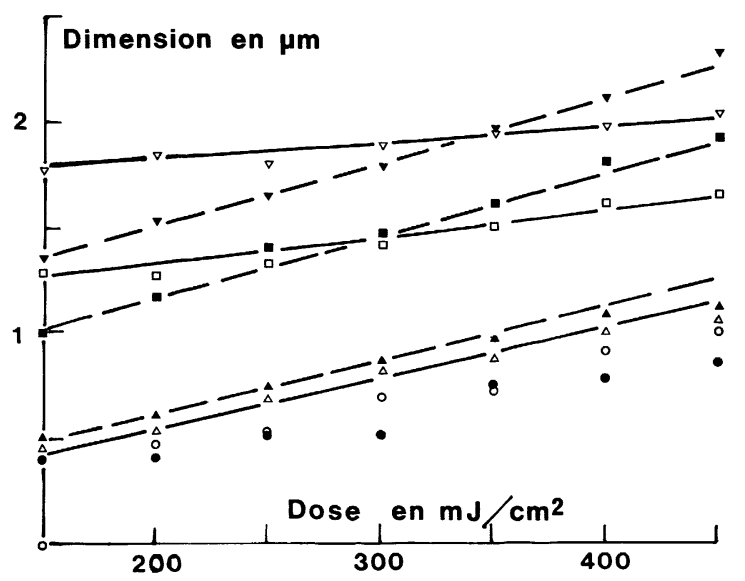

Fig. 2. - Variation des largeurs de motifs après développement en fonction de la dose d'insolation (dimensions de motifs 0,7 à $2 \mu \mathrm{m}$; intensités incidentes 122 et $304 \mathrm{~mW} / \mathrm{cm}^{2}$ ).

(O) $0,7 \mu \mathrm{m}-122 \mathrm{~mW} / \mathrm{cm}^{2}$;

(A) $1 \mu \mathrm{m}-122 \mathrm{~mW} / \mathrm{cm}^{2}$;

(घ) $1,6 \mu \mathrm{m}-122 \mathrm{~mW} / \mathrm{cm}^{2}$;

(V) $2 \mu \mathrm{m}-122 \mathrm{~mW} / \mathrm{cm}^{2}$;

(•) $0,7 \mu \mathrm{m}-304 \mathrm{~mW} / \mathrm{cm}^{2}$

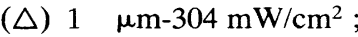

(ㅁ) $1,6 \mu \mathrm{m}-304 \mathrm{~mW} / \mathrm{cm}^{2}$;

(D) $2 \mu \mathrm{m}-304 \mathrm{~mW} / \mathrm{cm}^{2}$.

[The variation of linewidth after development as a function of the exposure dose (linewidth 0.7 to $2 \mu \mathrm{m}$; incident intensity 122 and $\left.304 \mathrm{~mW} / \mathrm{cm}^{2}\right)$.] 
- le taux de variation de dimension qui exprime la perte de côte en fonction de la dose d'insølation.

\section{Discussion :}

Les doses nominales sont reportées sur le tableau IV. Il apparaît que la dose nominale est plus rapidement atteinte pour les motifs de petite taille. Une tendance analogue est constatée par Utsugi [7, 8]. Ceci remet en question l'exacte correspondance entre ouverture dans le masque et taille des motifs révélés pour une même dose d'exposition lors d'insolations dont la durée n'excède pas quelques secondes.

Tableau IV. - Doses nominales en fonction de l'intensité incidente pour les différentes tailles de motifs en fonction de l'intensité incidente.

[Nominal doses versus the incident intensity for various pattern widthes.]

\begin{tabular}{|c|c|c|c|c|}
\hline${ }_{I_{0}}^{\text {Taille }}$ & $2 \mu \mathrm{m}$ & $1,6 \mu \mathrm{m}$ & $1 \mu \mathrm{m}$ & $0,7 \mu \mathrm{m}$ \\
\hline $122 \mathrm{~mW} / \mathrm{cm}^{2}$ & 430 & 410 & 395 & 350 \\
\hline $304 \mathrm{~mW} / \mathrm{cm}^{2}$ & 360 & 350 & 350 & 300 \\
\hline
\end{tabular}

Aucune tolérance d'exposition (plateau) n'apparaît quelle que soit l'intensité incidente. Les variations de largeurs de traits sont linéaires et croissent continuellement avec la dose d'insolation.

Deux traits caractéristiques peuvent être dégagés.

- La perte de contrôle de dimension est moins accentuée pour les fortes intensités incidentes (Tab. V) ; pour les motifs de $2 \mu \mathrm{m}$, elle est quatre fois plus importante à $122 \mathrm{~mW} / \mathrm{cm}^{2}$ qu'à $304 \mathrm{~mW} / \mathrm{cm}^{2}$.

- A intensité égale, la variation s'élève lorsque la taille des motifs diminue. Pour $304 \mathrm{~mW} / \mathrm{cm}^{2}$, elle double entre une taille nominale de $2 \mu \mathrm{m}$ et celle de

Tableau V. - Taux mesuré des variations de largeur de trait $\left(\mu \mathrm{m} / \mathrm{mJ} . \mathrm{cm}^{-2}\right)$.

[Measured rate of the linewidth variations.]

\begin{tabular}{|c|c|c|}
\hline Taille & $\begin{array}{c}\text { Intensité } \\
122 \mathrm{~mW} / \mathrm{cm}^{2}\end{array}$ & $\begin{array}{c}\text { Intensité } \\
304 \mathrm{~mW} / \mathrm{cm}^{2}\end{array}$ \\
\hline $0,7 \mu \mathrm{m}$ & $2,083 \times 10^{-3}$ & $1,32 \times 10^{-3}$ \\
\hline $1 \mu \mathrm{m}$ & $2,431 \times 10^{-3}$ & $2,222 \times 10^{-3}$ \\
\hline $1,6 \mu \mathrm{m}$ & $2,986 \times 10^{-3}$ & $9,033 \times 10^{-4}$ \\
\hline $2 \mu \mathrm{m}$ & $2,916 \times 10^{-3}$ & $6,944 \times 10^{-4}$ \\
\hline
\end{tabular}

REVUE DE PHYSIQUE APPLIQUEE - T 24, N` 12, DECEMBRE 1989
$0,7 \mu \mathrm{m}$. La même constatation est faite pour $122 \mathrm{~mW} / \mathrm{cm}^{2}$ si l'on exclut les mesures effectuées sur les deux motifs de dimension restreinte.

L'analyse des données bibliographiques confirme ces deux points (Tab. VI). Bien que nous ne disposions pas de l'intensité incidente utilisée par Tai et al. [3], un encadrement peut être effectué. Notre estimation la situe entre 2 à $10 \mathrm{~mW} / \mathrm{cm}^{2}$. La première valeur est obtenue en fixant la dose de saturation pour une épaisseur de $10 \mathrm{~nm} \mathrm{d'Ag} \mathrm{Ag}_{2} \mathrm{Se}$ sur le plateau dimensionnel, soit $200 \mathrm{~mJ} / \mathrm{cm}^{2}$ pour 100 secondes d'exposition ; la seconde, en évaluant à $1,2 \mathrm{~J} / \mathrm{cm}^{2}$ la dose nominale du motif (pour environ 120 secondes d'insolation).

Tableau VI. - Variations de largeur de trait extraites de la bibliographie.

[Linewidth variations from literature.]

\begin{tabular}{|c|c|c|c|}
\hline $\begin{array}{l}\text { Taille } \\
(\mu \mathrm{m})\end{array}$ & Intensité & $\begin{array}{c}\text { Variation } \\
\left(\mu \mathrm{m} / \mathrm{mJ} \mathrm{cm}^{-2}\right)\end{array}$ & Référence \\
\hline 0,6 & $0,4 \mathrm{~W} / \mathrm{cm}^{2}$ & $6,158 \times 10^{-3}$ & 8 \\
\hline 0,8 & $0,4 \mathrm{~W} / \mathrm{cm}^{2}$ & $4,506 \times 10^{-4}$ & 8 \\
\hline 1 & $0,4 \mathrm{~W} / \mathrm{cm}^{2}$ & $3,634 \times 10^{-4}$ & 8 \\
\hline 1,2 & $0,4 \mathrm{~W} / \mathrm{cm}^{2}$ & $2,980 \times 10^{-4}$ & 8 \\
\hline 1,25 & $2 \mathrm{~mW} / \mathrm{cm}^{2}(*)$ & $5 \times 10^{-3}$ & 3 \\
\hline 1,25 & $5 \mathrm{~mW} / \mathrm{cm}^{2}(*)$ & $2,00 \times 10^{-3}$ & 3 \\
\hline 1,25 & $10 \mathrm{~mW} / \mathrm{cm}^{2}(*)$ & $1,00 \times 10^{-3}$ & 3 \\
\hline
\end{tabular}

(*) Valeur estimée.

3.3 Simulation. - Le but de la simulation est d'estimer la perte de contrôle occasionnée par l'abaissement des intensités incidentes et l'environnement des motifs. Elle repose sur plusieurs hypothèses.

- L'absorption occasionnée par l'épaisseur de la couche de sensibilisation n'est pas de nature à modifier radicalement la distribution d'intensité transmise à l'interface. Ceci est particulièrement vrai dans notre cas de figure où l'épaisseur est mince et les intensités utilisées élevées.

- Par ailleurs, le séléniure de germanium étant opaque et le processus de photodopage localisé à la surface du dépôt, les variations dans les tailles des motifs développés doivent reproduire approximativement celles de l'image aérienne.

Autrement dit, notre simulation néglige la couche de sensibilisation et considère que le développement 
est anisotrope. Les effets introduits par la migration des atomes d'argent, diffusion latérale et absorption, sont globalisés et n'interviennent qu'au niveau de la dose seuil nécessaire à la survie des motifs.

3.3.1 Taille simulée des motifs. - Elle utilise la distribution d'intensité de l'image aérienne obtenue par le programme SAMPLE (version 1.6B). La figure 3 représente l'intensité (ou dose) normalisée pour différrentes tailles nominales de motifs L/S en bord d'ouverture du masque. La partie gauche correspond à la zone opaque; la partie droite représente l'intensité incidente dans la partie transparente pour un demi-motif. Lors du développement, seules les parties ayant bénéficié d'une insolation supérieure à la dose seuil demeureront. La largeur du motif est donc déterminée par l'abscisse

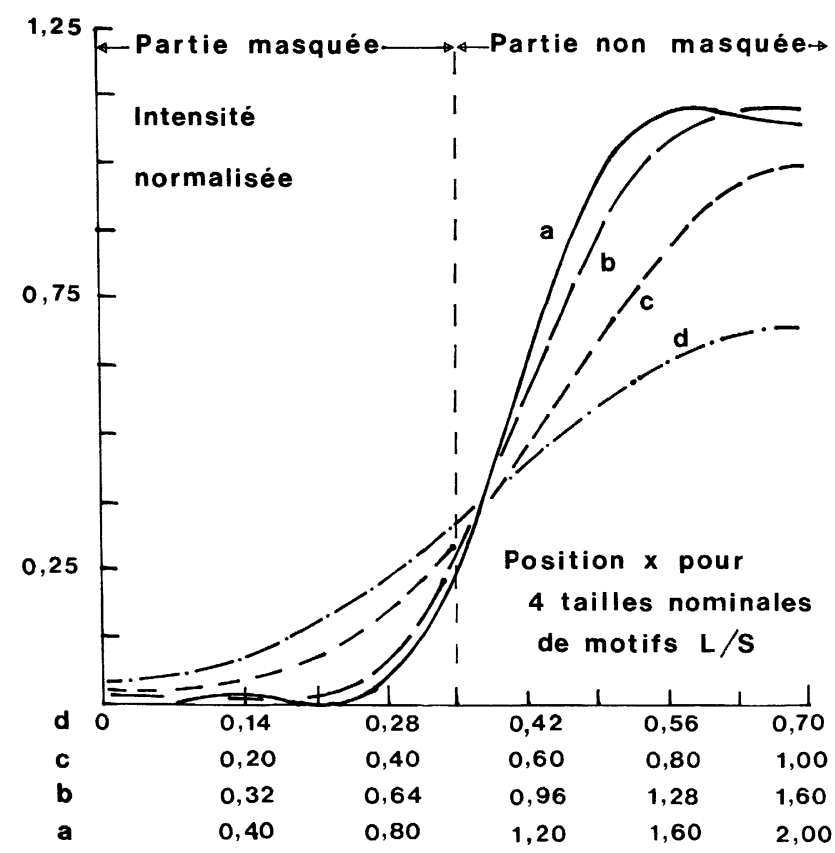

Fig. 3. - Distribution d'intensités normalisées simulées pour différentes largeurs de trait $(\mathrm{a}, \mathrm{b}, \mathrm{c}$, et $\mathrm{d}$ correspondent respectivement à des tailles nominales de motifs $\mathrm{L} / \mathrm{S}$ de $2,0,1,6,1,0$ et $0,7 \mu \mathrm{m})$.

[The distribution of simulated normalized intensities for various linewidthes.]

du point correspondant au rapport $E_{\text {SEUIL }} / E$. La figure 4 illustre l'accroissement de dimension d'un motif de $2 \mu \mathrm{m}$ lorsque la dose varie de 150 à $450 \mathrm{~mJ} / \mathrm{cm}^{2}$ en prenant une dose seuil de $150 \mathrm{~mJ} / \mathrm{cm}^{2}$. Les valeurs simulées des largeurs des motifs sont reportées dans les tableaux VII $(2 \mu \mathrm{m})$ et VIII $(1,6 \mu \mathrm{m})$.

3.3.2 Evolution de dimension en fonction de la dose seuil. - On constate que les variations de dimension sont accentuées lorsque la dose seuil s'élève (Fig. 5).

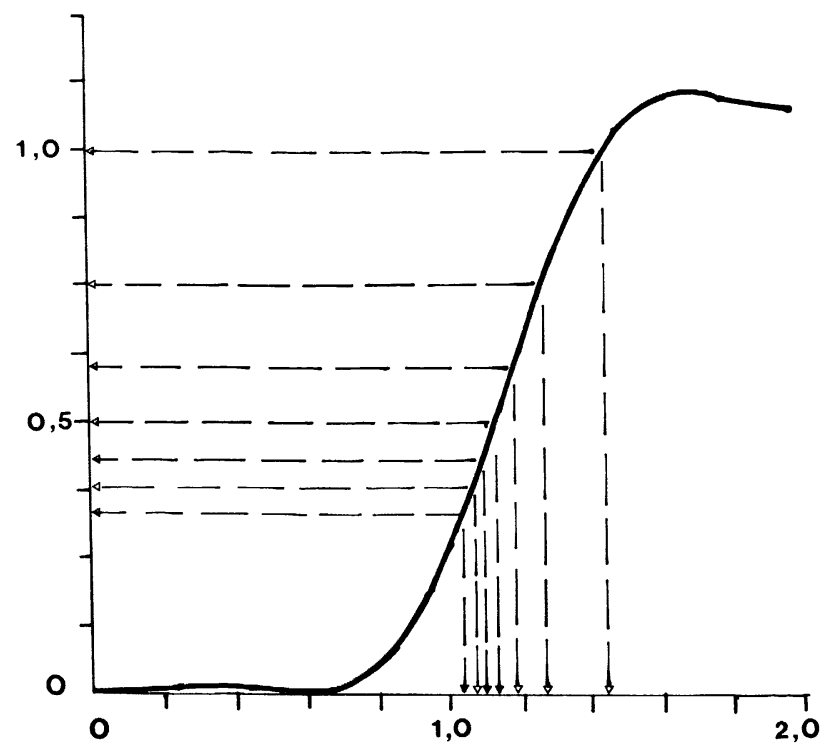

Fig. 4. - Evolution de la largeur d'un motif de taille nominale $2,0 \mu \mathrm{m}$ en fonction de la dose d'insolation (dose seuil $E_{\mathrm{s}}=150 \mathrm{~mJ} / \mathrm{cm}^{2}$ ).

[The variation of linewidth with $2.0 \mu \mathrm{m}$ nominal width as a function of the exposure dose (threshold dose $E_{\mathrm{s}}=$ $\left.150 \mathrm{~mJ} / \mathrm{cm}^{2}\right)$.]

Tableau VII. - Dimensions simulées pour un motif de $2 \mu \mathrm{m} \mathrm{L} / \mathrm{S}$ de taille nominale.

[Calculated values of linewidthes for a $2 \mu \mathrm{m} \mathrm{L/S}$ nominal pattern width.]

\begin{tabular}{|c|c|c|c|c|c|c|c|}
\hline & \multicolumn{6}{|c|}{$E_{\mathrm{s}}\left(\mathrm{mJ} / \mathrm{cm}^{2}\right)$} \\
\hline & & 30 & 50 & 70 & 100 & 130 & 150 \\
\hline \multirow{7}{*}{ 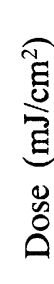 } & 150 & 2,062 & 1,908 & 1,786 & 1,575 & 1,316 & 1,110 \\
\hline & 200 & 2,162 & 1,975 & 1,924 & 1,722 & 1,569 & 1,468 \\
\hline & 250 & 2,211 & 2,075 & 2,000 & 1,843 & 1,721 & 1,620 \\
\hline & 300 & 2,261 & 2,125 & 2,050 & 1,924 & 1,797 & 1,747 \\
\hline & 350 & 2,311 & 2,175 & 2,100 & 1,975 & 1,873 & 1,797 \\
\hline & 400 & 2,360 & 2,213 & 2,139 & 2,020 & 1,924 & 1,848 \\
\hline & 450 & 2,385 & 2,250 & 2,139 & 2,123 & 1,975 & 1,924 \\
\hline
\end{tabular}

Une approximation linéaire permet d'estimer globalement la perte de contrôle qu'occasionne ce phénomène (Tab. IX). Celle-ci croît rapidement avec l'augmentation de la dose seuil mais reste sensiblement constante lorsque la taille du motif diminue.

3.3.3 Application au débit de dose. - La comparaison des valeurs simulées (Tab. IX) et mesurées (Tab. V) permet de situer les doses seuils nécessaires entraînant les variations enregistrées sur le système $\mathrm{Ag}_{2} \mathrm{Se} / \mathrm{GeSe}_{5,5}$ préparé par PECVD.

Pour une insolation effectuée avec une intensité incidente de $304 \mathrm{~mW} / \mathrm{cm}^{2}$, la dose seuil requise est 
Tableau VIII. - Dimensions simulées pour un motif de $1,6 \mu \mathrm{m} \mathrm{L} / \mathrm{S}$ de taille nominale.

[Simulated linewidthes from a $1.6 \mu \mathrm{m} \mathrm{L} / \mathrm{S}$ nominal pattern width.]

\begin{tabular}{|c|c|c|c|c|c|c|c|}
\hline & \multicolumn{6}{|c|}{$E_{\mathrm{s}}\left(\mathrm{mJ} / \mathrm{cm}^{2}\right)$} \\
\hline & & 30 & 50 & 70 & 100 & 130 & 150 \\
\hline \multirow{7}{*}{ 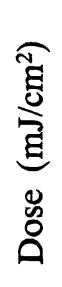 } & 150 & 1,662 & 1,521 & 1,361 & 1,155 & 0,982 & 0,725 \\
\hline & 200 & 1,771 & 1,640 & 1,509 & $\mid 1,337$ & 1,195 & 1,087 \\
\hline & 250 & 1,831 & 1,700 & 1,590 & 1,438 & 1,316 & 1,248 \\
\hline & 300 & 1,872 & 1,762 & 1,670 & 1,539 & 1,418 & 1,348 \\
\hline & 350 & 1,892 & 1,824 & 1,691 & 1,600 & 1,498 & 1,409 \\
\hline & 400 & 1,912 & 1,845 & 1,751 & 1,641 & 1,549 & 1,489 \\
\hline & 450 & 1,991 & 1,892 & 1,796 & 1,731 & 1,610 & 1,550 \\
\hline
\end{tabular}

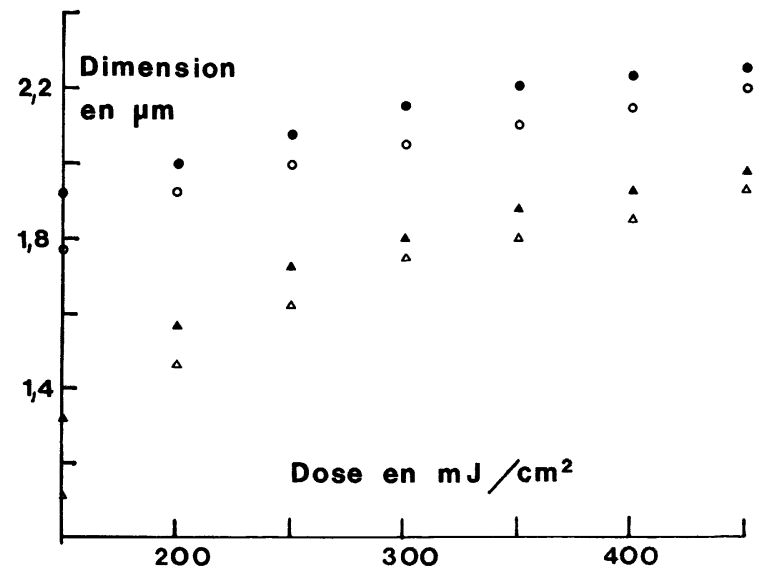

Fig. 5. - Evolution de dimension simulée d'un motif de taille nominale $2,0 \mu \mathrm{m}$ en fonction de la dose seuil $E_{\mathrm{s}}=50(\bullet), 70(\mathrm{O}), 130(\Delta)$ et $150(\Delta) \mathrm{mJ} / \mathrm{cm}^{2}$.

[Simulated linewidth variation for a $2.0 \mu \mathrm{m}$ nominal linewidth as a function of threshold dose.]

comprise dans une gamme de 30 à $70 \mathrm{~mJ} / \mathrm{cm}^{2}$ alors qu'une dose minimale de $130 \mathrm{~mJ} / \mathrm{cm}^{2}$ est nécessaire pour expliquer la perte de contrôle de dimension pour $122 \mathrm{~mW} / \mathrm{cm}^{2}$. Ces valeurs sont à rapprocher des valeurs expérimentales extraites des courbes de contraste (Tab. II) qui sont respectivement de $70-80 \mathrm{~mJ} / \mathrm{cm}^{2}$ pour $304 \mathrm{~mW} / \mathrm{cm}^{2}$ et de $120-130 \mathrm{~mJ} / \mathrm{cm}^{2}$ pour $122 \mathrm{~mW} / \mathrm{cm}^{2}$.

$\mathrm{Ce}$ modèle, très simplificateur, permet de confirmer les tendances enregistrées sur les mesures. En effet, il apparaît que la perte de contrôle est accentuée lors d'insolation à basse intensité. Il confirme la corrélation qui existe entre ce fait expérimental et les doses seuils réellement mesurées.

3.3.4 Application à l'environnement. - Cette analyse en terme d'augmentation de dose seuil peut aussi expliquer les variations de côtes plus brutales des motifs de petites tailles lors d'insolation à intensité constante. En effet, le contraste d'exposition sur ces motifs de tailles réduites est minimal. De plus, lorsque la dose d'insolation s'accroît, la longueur de diffusion des atomes d'argent dépasse rapidement la largeur de leurs zones floues (la zone floue ou zone de transition peut être estimée grâce aux paramètres machines par la relation $T_{\mathrm{W}}=\lambda / 2 O N$, avec $O N=$ ouverture numérique). Ceci contribue à une amélioration de l'absorption de la couche de sensibilisation. Elle est d'autant plus efficace que la taille des motifs est restreinte. C'est pourquoi ces derniers atteindront le seuil développement pour des doses sensiblement plus élevées que celles requises pour l'insolation de motifs plus larges. Ainsi, il est logique de penser que la diminution de la taille nominale s'accompagne d'une élévation des doses minimales nécessaires à la survie des motifs (doses seuils). Ceci implique, parallèlement, une dégradation du contrôle de dimension. Cette interprétation s'applique aussi bien à nos mesures qu'à celles obtenues par Utsugi.

3.3.5 Limitations du modèle. - La principale restriction est qu'il ne peut pas rendre compte de manière exacte de l'évolution des dimensions des motifs pour des doses d'exposition croissantes. En effet, il revient à assimiler l'image aérienne à l'image latente. La taille du motif est alors déterminée en supposant que le seuil de développement est atteint pour des doses plus ou moins élevées. Ces doses seuils variables tiennent compte des conditions d'insolation et de l'environnement. En réalité,

Tableau IX. - Perte de contrôle de dimension (en $\left.\mu \mathrm{m} / \mathrm{mJ} . \mathrm{cm}^{-2}\right)$ en fonction de la dose seuil $\left(e n \mathrm{~mJ} / \mathrm{cm}^{2}\right)$. [Loss of linewidth control $\left(\mu \mathrm{m} / \mathrm{mJ} . \mathrm{cm}^{-2}\right)$ versus the threshold dose $\left(\mathrm{mJ} / \mathrm{cm}^{2}\right)$.]

\begin{tabular}{|c|c|c|c|c|c|c|}
\cline { 2 - 7 } \multicolumn{1}{c|}{} & \multicolumn{6}{c|}{$E_{\mathrm{s}}\left(\mathrm{mJ} / \mathrm{cm}^{2}\right)$} \\
\hline $2 \mu \mathrm{m}$ & $9,23 \times 10^{-4}$ & $9,77 \times 10^{-4}$ & $1,15 \times 10^{-3}$ & $1,57 \times 10^{-3}$ & $1,88 \times 10^{-3}$ & $2,33 \times 10^{-3}$ \\
\hline $1,6 \mu \mathrm{m}$ & $9,4 \times 10^{-4}$ & $1,06 \times 10^{-3}$ & $1,24 \times 10^{-3}$ & $1,65 \times 10^{-3}$ & $1,94 \times 10^{-3}$ & $2,36 \times 10^{-3}$ \\
\hline
\end{tabular}


l'image latente n'est pas la reproduction de l'image aérienne. La diffusion latérale et l'absorptivité de la couche contribuent à accentuer les écarts existants entre les deux courbes. La dimension des motifs doit être simulée en prenant un seuil de développement constant que l'on applique sur la distribution en ions argent photodopés (image latente) variable avec le temps d'insolation. Il est donc normal que nos valeurs simulées divergent des tailles réellement mesurées. De même, notre simulation s'avère insuffisante pour rendre compte de l'existence d'un éventuel plateau dimensionnel.

\section{Conclusion.}

Il existe plusieurs modèles pour expliquer la formation de l'image latente sur les chalcogénures. L'interprétation basée sur le concept de photodopage demeure la plus naturelle. Nous pensons que la taille des motifs est effectivement déterminée par la quantité locale d'argent photodopé dans la matrice.

Après l'analyse des critères d'apparition des phénomènes associés à la migration des ions argent, il nous a paru nécessaire d'étudier l'influence du temps d'exposition sur la lithographie des chalcogénures. Il ressort des expériences que nous avons menées, qu'un abaissement de l'intensité incidente procure une meilleure sélectivité au système. Ce fait est conciliable avec une augmentation de la quantité d'argent photodopé au centre des motifs et est en relation avec l'accroissement de la longueur de diffusion des atomes d'argent dans la couche de sensibilisation. Toutefois, les implications sur le contraste, et par conséquent sur la résolution du système, restent faibles.

En contrepartie, nous montrons que l'utilisation d'un faible débit de dose s'accompagne d'une élévation des doses seuils. Ceci induit une accentuation de la perte de contrôle de dimension. Cette analyse est confortée par une simulation que nous avons développée dans le but d'estimer la variation globale des largeurs de traits en fonction de la dose d'exposition. Celle-ci, reposant sur des hypothèses très simplificatrices, ne permet pas une représentation exacte du processus de formation de l'image latente. Elle confirme toutefois la corrélation qui existe entre les mesures des doses seuils et les pertes de contrôle de dimension observées. Les tendances enregistrées sont une dégradation accentuée de la largeur des traits reproduits avec l'abaissement de l'intensité incidente d'une part, et de la taille nominale des motifs d'autre part. Une élévation de la dose seuil en est à l'origine, mais cette élévation peut être corrigée par l'emploi d'un autre révélateur plus « dur». Le modèle reste limité et ne peut prendre en compte les variations locales de photodopage qui se traduisent par l'existence d'un plateau lorsqu'on représente la dimension des motifs en fonction du temps d'exposition lors d'insolations prolongées.

\section{Bibliographie}

[1] Eranian A. et Dubois J. C., Rev. Techn. Thomson CSF 19 (1987) 95.

[2] Tai K. L., Vadimsky R. G. et Lamberti V. E., ECS. Ext. Abst. 80 (1980) 826.

[3] TaI K. L., Vadimsky R. G., Kemmerer C. T., Wagner J. S., Lamberti V. E. et Timko A. G., J. Vac. Sci. Technol. 17 (1980) 1169.

[4] Tai K. L., Ong E., Vadimsky R. G., Kemmerer C. T. et Bridenbaugh P. M., Proc. Symp. Inorg. Resists ECS-82-9 (1982) p. 49.

[5] Lamberti V. E., Vincent S. M., Kemmerer C. T. et VAdimsky R. G., Proc. Symp. Inorg. Resists ECS-82-9 (1982) p. 191.

[6] Ong E., Tai K. L., Vadimsky R. G. et Kemmerer C. T., Proc. SPIE 394 (1983) 39.

[7] Utsugi Y., Doken M. et YoshiKawa A., Electrochem. Soc. Ext. Abst. 84 (1984) 774.

[8] Utsugi Y., Yoshikawa A. et Katayawa T., Microelectron. Eng. 2 (1984) 281.

[9] Leung W., Neureuther A. R. et Oldham W. G., Proc. SPIE 394 (1983) 134.

[10] Leung W., Neureuther A. R. et Oldham W. G., J. Vac. Sci. Technol B 3-1 (1985) 310.
[11] Leung W., Neureuther A. R. et Oldham W. G., IEEE Trans. Elec. Devices ED 33 (1986) 173.

[12] Funakoshi W., Zembutsu S. et Kasai T., Jpn J. Appl. Phys. Lett. 20 (1981) 649.

[13] Funakoshi N., Fujimori S., Zembutsu J. et Kasai T., Jpn J. Appl. Phys. Lett. 21 (1982) 40.

[14] Tai K. L., Jpn J. Appl. Phys. Lett. 21 (1982) 300.

[15] Funakoshi N. et Zembutsu J., Jpn J. Appl. Phys. Lett. 21 (1982) 300.

[16] Nagai H., Yoshikawa A., Toyoshima Y., OCHI O. et Mizushima Y., Appl. Phys. Lett. 28-3 (1976) 145.

[17] Yoshikawa A., Ochi O., Nagai H. et Mizushima Y., Appl. Phys. Lett. 29-10 (1976) 677.

[18] Yoshikawa A., OCHI O., Nagai H. et Mizushima Y., Appl. Phys. Lett. 31-3 (1977) 161.

[19] Yoshikawa A., Ochi O. et Mizushima Y., Appl. Phys. Lett. 36-1 (1980) 107.

[20] Hugett P. G., Frick F. et Lehmann H. W., Appl. Phys. Lett. $42-7$ (1983) 592. 